\title{
Gut microbiota influences liver disease
}

\begin{abstract}
Scientists reporting in Nature have identified a key factor that causes the progression of a usually asymptomatic metabolic liver condition to chronic inflammatory liver disease. It depends on the composition of your gut microbiota.

Only $20 \%$ of individuals with nonalcoholic fatty liver disease (NAFLD) develop chronic hepatic inflammation known as non-alcoholic steatohepatitis (NASH). To investigate what determines this disease progression, Flavell and colleagues used a mouse model of NASH, which could be induced by feeding wild-type animals a methionine- and choline-deficient (MCD) diet. Given its role in the generation of the pro-inflammatory cytokines interleukin-1 $\beta$ (IL-1 $\beta$ )
\end{abstract}

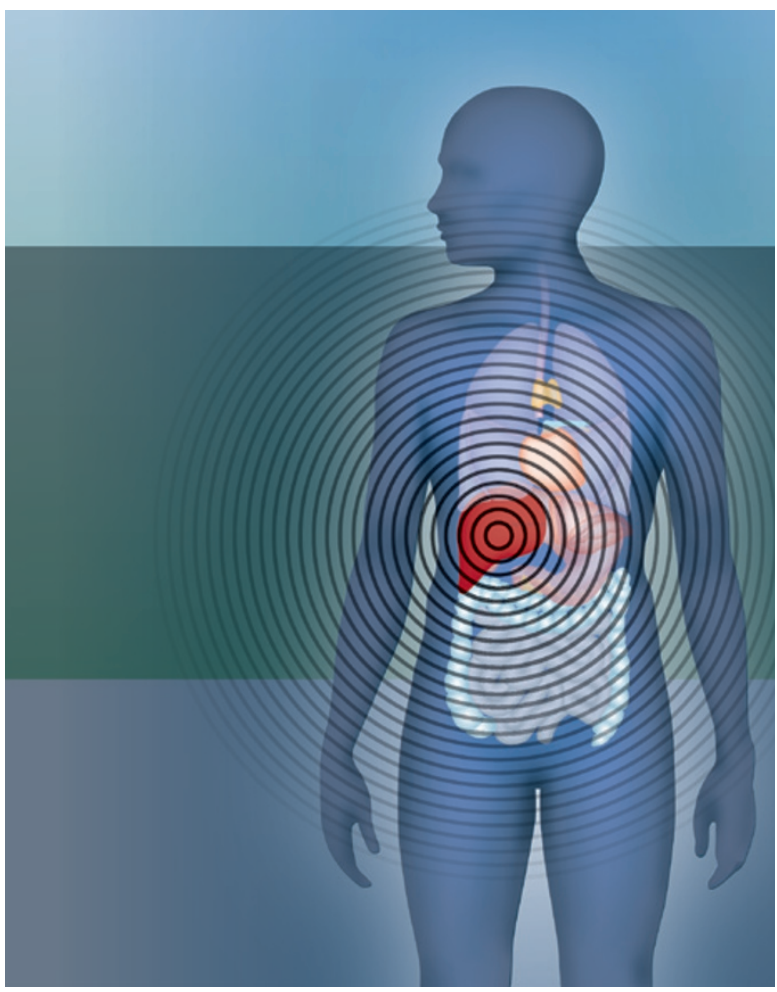

and IL-18, the inflammasome was proposed as a likely factor in disease progression. Indeed, mice that lacked functional inflammasomes, owing to genetic deletion of $A s c$ (which encodes an inflammasome component) or Casp1 (which encodes the inflammasome-associated enzyme caspase 1), developed more severe liver disease after the MCD diet than wild-type mice. Similarly, IL-18-deficient mice, but not IL-1 receptor-deficient mice, developed severe NASH when fed the MCD diet, suggesting a key role for IL-18 in negative regulation of disease progression. Analyses of other mutant mice revealed a role for aberrant function of the NLRP3 (NOD-, LRRand pyrin domain-containing 3 ) inflammasome in driving liver disease progression.

The authors have recently shown that inflammasomes are sensors and regulators of the colonic microbiota, and that when they are disrupted the composition of the gut microbiota is altered. Could this altered composition influence events in the liver? To test this, the authors housed wild-type mice together with $\mathrm{Asc}^{-1-}$, Casp $\mathrm{I}^{-{ }_{-}}$, $\mathrm{Ill1}^{-/-}$or $\mathrm{Nlrp}^{3^{-/}}$mice before the induction of NASH with the MCD diet. Remarkably, co-housing was sufficient to cause NASH in the wild-type mice, and their disease was comparable to that of the inflammasomedeficient mice. This indicated that the transmissible colonic gut microbiota in inflammasome-deficient mice is a major contributor to NASH severity.

The gut and the liver are connected via the portal circulation, and so the authors next investigated whether bacterium-derived products that leak into the blood from the gut might activate Toll-like receptors (TLRs) expressed in the liver. In agreement with this hypothesis, mice that lacked TLR signalling capability

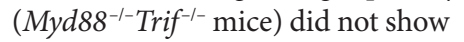
enhanced NASH after being cohoused with $\mathrm{Asc}^{-1-}$ mice and fed the MCD diet. Further studies showed that TLR4 and TLR9 are involved in mediating microbiota-induced liver disease. TLR signalling in the liver led to the production of tumour necrosis factor, which was found to be responsible for mediating the downstream hepatotoxic effects.

NAFLD is commonly associated with obesity and insulin resistance, but these factors are not necessary for MCD diet-induced NASH in mice. To more closely model these metabolic abnormalities, the authors studied leptin receptor-deficient $(d b / d b)$ mice, which develop many metabolic defects, including NAFLD. When $d b / d b$ mice were housed with $A s c^{-1-}$ mice, but not when they were housed with wild-type mice, they developed marked liver inflammation and showed dramatic weight gain. An association between altered gut microbiota and metabolic abnormalities (including weight gain, glucose intolerance and liver disease) was also observed when $\mathrm{Asc}^{-1-}$ mice, but not wild-type mice, were fed a high-fat diet. Together, these findings suggest that changes in the composition of the microbiota as a result of inflammasome defects can cause various metabolic abnormalities and trigger the progression of inflammatory liver disease.

Lucy Bird

ORIGINAL RESEARCH PAPER Henao-Mejia, J.et al. Inflammasome-mediated dysbiosis regulates progression of NAFLD and obesity. Nature $\mathbf{4 8 2}$, 179-185 (2012) 